\title{
An energy-based approach to estimate seismic attenuation due to wave-induced fluid flow in heterogeneous poroelastic media
}

\author{
Santiago G. Solazzi, ${ }^{1}$ J. Germán Rubino, ${ }^{2,}{ }^{*}$ Tobias M. Müller, ${ }^{3}$ Marco Milani, ${ }^{2}$ \\ Luis Guarracino ${ }^{1}$ and Klaus Holliger ${ }^{2}$ \\ ${ }^{1}$ CONICET, Facultad de Ciencias Astronómicas y Geofísicas, Universidad Nacional de La Plata, B1900 La Plata, Argentina. \\ E-mail:ssolazzi@fcaglp.unlp.edu.ar \\ ${ }^{2}$ Applied and Environmental Geophysics Group, Institute of Earth Sciences, University of Lausanne, CH-1015 Lausanne, Switzerland \\ ${ }^{3}$ CSIRO Energy, Perth, WA 6151, Australia
}

Accepted 2016 August 4. Received 2016 August 3; in original form 2015 October 8

\begin{abstract}
SUMMAR Y
Wave-induced fluid flow (WIFF) due to the presence of mesoscopic heterogeneities is considered as one of the main seismic attenuation mechanisms in the shallower parts of the Earth's crust. For this reason, several models have been developed to quantify seismic attenuation in the presence of heterogeneities of varying complexity, ranging from periodically layered media to rocks containing fractures and highly irregular distributions of fluid patches. Most of these models are based on Biot's theory of poroelasticity and make use of the assumption that the upscaled counterpart of a heterogeneous poroelastic medium can be represented by a homogeneous viscoelastic solid. Under this dynamic-equivalent viscoelastic medium (DEVM) assumption, attenuation is quantified in terms of the ratio of the imaginary and real parts of a frequency-dependent, complex-valued viscoelastic modulus. Laboratory measurements on fluid-saturated rock samples also rely on this DEVM assumption when inferring attenuation from the phase shift between the applied stress and the resulting strain. However, whether it is correct to use an effective viscoelastic medium to represent the attenuation arising from WIFF at mesoscopic scales in heterogeneous poroelastic media remains largely unexplored. In this work, we present an alternative approach to estimate seismic attenuation due to WIFF. It is fully rooted in the framework of poroelasticity and is based on the quantification of the dissipated power and stored strain energy resulting from numerical oscillatory relaxation tests. We employ this methodology to compare different definitions of the inverse quality factor for a set of pertinent scenarios, including patchy saturation and fractured rocks. This numerical analysis allows us to verify the correctness of the DEVM assumption in the presence of different kinds of heterogeneities. The proposed methodology has the key advantage of providing the local contributions of energy dissipation to the overall seismic attenuation, information that is not available when attenuation is retrieved from methods based on the DEVM assumption. Using the local attenuation contributions we provide further insights into the WIFF mechanism for randomly distributed fluid patches and explore the accumulation of energy dissipation in the vicinity of fractures.
\end{abstract}

Key words: Seismic attenuation; Computational seismology; Theoretical seismology; Acoustic properties.

\section{INTRODUCTION}

Whenever a seismic wave propagates through a fluid-saturated porous rock that contains heterogeneities in the mesoscopic scale range, that is, heterogeneities that are much larger than the typical pore size but much smaller than the predominant wavelengths, local

\footnotetext{
* Now at: Department of Earth Sciences, University of Western Ontario, London, ON N6A5B7, Canada.
}

gradients in the pore fluid pressure arise. These pressure gradients, which are due to the uneven response of the heterogeneities to the stresses associated with the passing seismic wavefield, induce viscous fluid flow and thus energy dissipation through internal friction. This attenuation mechanism scales with the compressibility contrast between the heterogeneities and their embedding background. Correspondingly, environments characterized by patchy saturation as well as fractured media represent particularly prominent scenarios where attenuation due to wave-induced fluid flow (WIFF) is expected to be predominant (Müller et al. 2010). 
White (1975) and White et al. (1975) were the first to propose fluid flow at the mesoscopic scale as an attenuation mechanism of seismic waves propagating in a water-saturated porous matrix containing spherical gas pockets and in a porous periodically layered medium alternately saturated with gas and water. Using a more sophisticated approach based on Biot's (1962) poroelastic equations, Dutta \& Odé (1979a,b) presented a theory to compute seismic attenuation and dispersion for White's (1975) model. The case of irregular gas patches was treated by Johnson (2001), who analysed the dynamic undrained bulk modulus of porous rocks characterized by patchy saturation using branching functions. All these models have the advantage of being based on relatively simple semi-analytical expressions, which allow for a straightforward assessment of the influence of various physical parameters on the seismic response. Due to the limitations of these and other approaches to rather simple model geometries and particular physical properties of the heterogeneities composing the probed medium, several authors considered numerical upscaling procedures to handle more complex scenarios.

Numerical upscaling methods are based on the application of oscillatory creep or relaxation tests to representative samples containing mesoscale heterogeneities. When subjected to such numerical tests, the sample exhibits a distinct response in the frequency domain, which allows to determine an equivalent complex-valued and frequency-dependent viscoelastic modulus. Masson \& Pride (2007) applied bulk compression and pure shear to a sample containing mesoscale heterogeneities and determined the equivalent viscoelastic response of the medium from the resulting average strain. Rubino et al. (2009) presented a finite-element procedure in the space-frequency domain to simulate compressibility and shear tests to obtain the equivalent complex plane-wave and shear moduli for highly heterogeneous samples. Wenzlau et al. (2010) and Quintal et al. (2011) inferred the attenuation and dispersion characteristics of the probed samples through numerical time-domain strain experiments based on Biot's (1941) quasi-static consolidation equations. Similar concepts are also employed in the context of corresponding laboratory experiments. For instance, Spencer (1981), Takei et al. (2011) and Tisato \& Quintal (2013) obtained seismic attenuation estimates in laboratory experiments by measuring the phase shift between the applied stress and the resulting strain in partially saturated rock samples.

All the above-mentioned works rely on the dynamic-equivalent viscoelastic medium (DEVM) assumption. That is, the heterogeneous porous rock sample subjected to a stress or a strain field can be represented by an equivalent homogeneous viscoelastic solid that exhibits the same overall response as a function of frequency. Under this assumption, the volume averages of the stress and strain fields in response to a specific set of boundary conditions allow to infer an equivalent complex-valued frequency-dependent undrained plane-wave modulus or an equivalent shear modulus. The inverse quality factor $1 / Q^{\mathcal{D}}$, which quantifies the overall seismic attenuation of the probed sample, is then given by the ratio between the imaginary and the real parts of the corresponding complex modulus (e.g. Borcherdt 2009)

$$
\frac{1}{Q_{p}^{\mathcal{D}}(\omega)}=\frac{\Im\left\{\bar{M}_{c}(\omega)\right\}}{\Re\left\{\bar{M}_{c}(\omega)\right\}}, \quad \frac{1}{Q_{s}^{\mathcal{D}}(\omega)}=\frac{\Im\left\{\bar{\mu}_{c}(\omega)\right\}}{\Re\left\{\bar{\mu}_{c}(\omega)\right\}},
$$

where $\omega$ is the angular frequency. The equivalent plane-wave and shear moduli, $\bar{M}_{c}$ and $\bar{\mu}_{c}$, are used in the case of $P$ - or $S$-wave attenuation, $1 / Q_{p}^{\mathcal{D}}$ and $1 / Q_{s}^{\mathcal{D}}$, respectively. The resulting equivalent viscoelastic solids have been employed by some authors to perform numerical simulations of seismic wave propagation considering WIFF effects (e.g. Rubino et al. 2011; Sidler et al. 2013) or to compare the resulting attenuation and phase velocity curves with those corresponding to specific viscoelastic models (Picotti et al. 2010). However, a rigorous analysis regarding the correctness of using viscoelasticity theory to compute attenuation due to WIFF at mesoscopic scales is still missing in the literature. A recent effort in this direction was published by Jänicke et al. (2015), who derived an upscaling procedure based on an extension of the Hill-Mandel criterion (Hill 1963, 1972). The main reason why it is important to explore the DEVM assumption is that, regardless of the physics governing at the local scale and of the imposed boundary conditions, it is always possible to define equivalent elastic moduli in terms of volume averages of the stress and strain fields in response to numerical oscillatory tests. However, it is not guaranteed that such equivalent moduli adequately represents the overall behaviour of the original heterogeneous sample with respect to both the dissipated energy and the elastic strain energy. Failure to do so would imply that the seismic attributes extracted from such equivalent elastic moduli would not be truly representative of the behaviour of the heterogeneous sample. In the case of elastic or viscoelastic composites, the equivalence between elastic moduli defined in terms of energy and those obtained from relations between average stress and average strain has been established (Hill 1963; Hashin 1964). However, in this work the original heterogeneous porous sample is locally governed by fluid-pressure diffusion, whereas under the DEVM assumption, the upscaled behaviour is controlled by linear viscoelasticity. Consequently, the equivalence between approaches based on the DEVM assumption and those based on energy-related considerations in the original heterogeneous porous rock is not obvious. To test and corroborate the correctness of the DEVM assumption, alternative approaches fully rooted in the theoretical framework of poroelasticity should be considered.

Based on the concept of energy dissipation, the inverse quality factor can be defined as (e.g. O'Connell \& Budiansky 1978; Aki \& Richards 1980)

$\frac{1}{Q^{\mathcal{M}}(\omega)}=\frac{\langle\Delta \mathcal{P}(\omega)\rangle}{\omega \mathcal{W}_{\max }(\omega)}$,

where $\langle\Delta \mathcal{P}\rangle$ is the dissipated power averaged over one wave cycle and $\mathcal{W}_{\max }$ is the peak strain energy stored in the probed sample during the same cycle. Since WIFF is a dissipation mechanism, eq. (2) implies that attenuation can be quantified by computing $\langle\Delta \mathcal{P}\rangle$ and $\mathcal{W}_{\max }$ within the poroelastic framework without making any assumptions regarding the overall response of the probed sample. This expression of the inverse quality factor thus can be employed to verify the DEVM assumption.

An alternative definition for the inverse quality factor proposed by O'Connell \& Budiansky (1978) is

$$
\frac{1}{Q^{\mathcal{A}}(\omega)}=\frac{\langle\Delta \mathcal{P}(\omega)\rangle}{2 \omega\langle\mathcal{W}(\omega)\rangle},
$$

where $\langle\mathcal{W}\rangle$ denotes the strain energy of the sample averaged over one cycle. Please note that in the case of linear viscoelasticity, it can be shown that $\mathcal{W}_{\max } \simeq 2\langle\mathcal{W}\rangle$ for $Q^{\mathcal{M}} \gtrsim 10$ (Bourbié et al. 1987 ) and, consequently, eqs (2) and (3) are equivalent under these conditions. It can also be shown that, for this kind of viscoelastic materials, eq. (1) is compatible with eq. (3), that is, $Q^{\mathcal{A}}$ and $Q^{\mathcal{D}}$ are identical (O’Connell \& Budiansky 1978; Masson \& Pride 2014).

To our knowledge, mesoscopic WIFF studies were so far based on the DEVM assumption (eq. 1), and the equivalence of such approaches with the two energy-based formulations presented in eqs (2) and (3) has never been explored for heterogeneous 
poroelastic materials. In this work, we present a novel methodology to compute seismic attenuation due to WIFF at mesoscopic scales that is fully rooted in the theoretical framework of poroelasticity. To do so, we quantify the power loss and the strain energy of a heterogeneous poroelastic sample subjected to a numerical oscillatory relaxation test and obtain the inverse quality factor using eqs (2) and (3). We compare the results with those computed based on the DEVM assumption for two relevant cases: patchy saturation and fractured rocks. Finally, in the framework of the proposed energybased approach, we define and analyse the local contributions to the overall seismic attenuation, which constitute a new tool that may help to better understand the WIFF phenomenon.

\section{THEORY}

\subsection{Poroelasticity equations and numerical simulations of the displacement fields}

In order to explore WIFF effects, we solve Biot's (1941) quasi-static poroelasticity equations. The reasoning behind this is that, for frequencies well below Biot's characteristic frequency, WIFF process is controlled by fluid-pressure diffusion and hence inertial effects can be neglected. The total stress equilibrium and Darcy's law are then to be fulfilled simultaneously, which in space-frequency domain yields

$\nabla \cdot \sigma=0$

$\nabla p_{f}=-i \omega \frac{\eta}{\kappa} \boldsymbol{w}$

where $\sigma$ represents the total stress tensor, $p_{f}$ the fluid pressure, $\boldsymbol{w}$ the relative fluid-solid displacement, $\kappa$ the permeability and $\eta$ the viscosity of the pore fluid.

Eqs (4) and (5) are coupled through the stress-strain relations (Biot 1962)

$\boldsymbol{\sigma}=2 \mu_{m} \boldsymbol{\epsilon}+\boldsymbol{I}\left(\lambda_{c} \nabla \cdot \boldsymbol{u}-\alpha M \zeta\right)$,

$p_{f}=-\alpha M \nabla \cdot \boldsymbol{u}+M \zeta$,

where $\boldsymbol{I}$ is the identity matrix, $\boldsymbol{u}$ the solid displacement and $\zeta=$ $-\nabla \cdot \boldsymbol{w}$ a measure of the local change in the fluid content. The strain tensor is given by $\boldsymbol{\epsilon}=\frac{1}{2}\left(\nabla \boldsymbol{u}+(\nabla \boldsymbol{u})^{\mathrm{T}}\right)$, with $\mathrm{T}$ denoting the transpose. The poroelastic parameters $\alpha, M$ and $\lambda_{c}$ are given by (e.g. Rubino et al. 2009)

$\alpha=1-\frac{K_{m}}{K_{s}}$

$M=\left(\frac{\alpha-\phi}{K_{s}}+\frac{\phi}{K_{f}}\right)^{-1}$,

$\lambda_{c}=K_{m}+\alpha^{2} M-\frac{2}{3} \mu_{m}$,

with $\phi$ denoting the porosity, $\mu_{m}$ the shear modulus of the bulk material, which is equal to that of the dry frame and $K_{f}, K_{s}$ and $K_{m}$ the bulk moduli of the fluid phase, the solid grains and the dry matrix, respectively.

To compute the fluid and solid displacement fields associated with a passing monochromatic $P$ wave, we extend the approach of Rubino et al. (2009, 2014). That is, we consider a rectangular rock sample $\Omega$ containing a distribution of mesoscale heterogeneities subjected to a time-harmonic vertical solid displacement of the form $-\Delta u e^{i \omega t}$ (a)

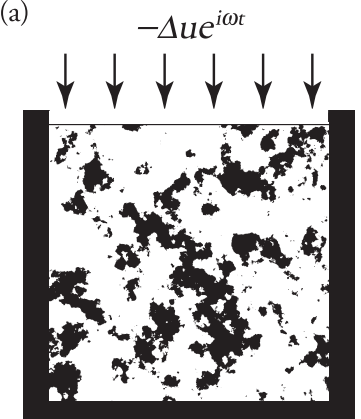

(b)

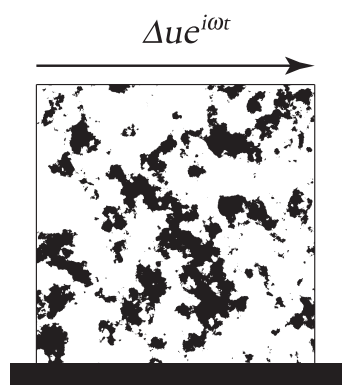

Figure 1. Schematic illustration of the numerical relaxation tests employed for determining (a) $P$-wave and (b) $S$-wave properties. An oscillatory displacement $\Delta u$ is applied along the top boundary of the samples in order to emulate the stress and strain fields associated with the passing wavefield.

along its top boundary (Fig. 1a). No tangential forces are imposed on the boundaries of the sample and the solid is neither allowed to move vertically on the bottom boundary nor to have horizontal displacements on the lateral boundaries. The fluid is neither allowed to flow into the sample nor out of it. To consider the case of a passing $S$ wave, we impose a solid horizontal displacement of the form $\Delta u e^{i \omega t}$ at the top boundary of the sample (Fig. 1b). No normal forces are imposed on the boundaries of the sample, whereas vertical displacements at the lateral boundaries and horizontal displacements at the bottom boundary are not allowed. Again, fluid is not allowed to flow into or out of the sample.

A finite-element procedure is employed to solve Biot's (1941) equations under the previously described boundary conditions, which are formally presented in Appendix. We use bilinear functions to approximate the solid displacement vector and a closed subspace of the vector part of the Raviart-Thomas-Nedelec space of zero order for representing the relative fluid displacement (Raviart \& Thomas 1977; Nedelec 1980). These displacement fields allow us to compute $P$ - and $S$-wave attenuation, either following an approach based on the DEVM assumption or an energy-based strategy. Please note that, in presence of heterogeneities having elongated shapes and preferential orientations, the probed sample will be effectively anisotropic. In this situation, the methodologies considered in this work only provide estimates of the characteristics of seismic waves propagating in the vertical direction. To obtain the seismic responses for arbitrary directions of wave propagation all the components of an equivalent complex-valued frequency-dependent stiffness matrix need to be determined. This, however, is outside the scope of this work.

It is important to remark here that, for the sake of simplicity in the description of the results and for numerical convenience, we have opted for 2-D samples. However, the proposed technique can be readily generalized for corresponding 3-D scenarios. It should also be noted that as we solve the equations in the space-frequency domain we employ complex notation to express the applied boundary conditions. Nevertheless, if we are interested in the behaviour of a given variable in the space-time domain, we consider its corresponding real part.

\subsection{Energy-based approach to quantify seismic attenuation}

The behaviour of the heterogeneous sample subjected to the numerical relaxation experiments described above allows to obtain energy-based attenuation estimates through eqs (2) and (3). To do 
so, the dissipated power and the strain energy stored within the sample need to be computed. In the following, we describe how these quantities can be obtained.

\subsubsection{Average dissipated power}

The dissipated power quantifies the energy being lost in the system per unit time. In the case of fluid-saturated porous materials, energy dissipation is due to viscous friction and, consequently, its magnitude strongly depends on the average relative motion between the fluid and the solid. For a monochromatic wave of angular frequency $\omega$, the local contribution to the dissipated power as a function of time can be expressed as (Biot 1962; Bourbié et al. 1987)

$\left.\mathrm{d} \mathcal{P}(t)\right|_{\omega}=\hat{\dot{\boldsymbol{w}}}^{\mathrm{T}} \boldsymbol{K} \hat{\dot{\boldsymbol{w}}} \mathrm{dV}$,

where $\boldsymbol{K}=\eta / \kappa \boldsymbol{I}$, and $\mathrm{dV}$ is the volume of the elementary region where the contribution is computed. As we solve the equations in the space-frequency domain, whereas the relative fluid velocity $\hat{\dot{w}}$ in eq. (11) is expressed in the space-time domain, the notation $\hat{a}=$ $\Re\left(a e^{i \omega t}\right)$ is chosen to express the corresponding time-dependent parameter, with $a$ being a given complex-valued variable.

Using averaging properties of time-harmonic complex-valued variables (e.g. Rubino et al. 2006) and taking into account that $\dot{\boldsymbol{w}}=i \omega \boldsymbol{w}$, it can be shown that the total dissipated power averaged over one wave cycle of oscillation is given by (Johnson 2001)

$\langle\Delta \mathcal{P}(\omega)\rangle=\left.\frac{\omega}{2 \pi} \int_{\Omega} \int_{0}^{2 \pi / \omega} \mathrm{d} \mathcal{P}(\mathrm{t})\right|_{\omega} \mathrm{dtdV}=\frac{\omega^{2}}{2} \int_{\Omega} \frac{\eta}{\kappa}\|\boldsymbol{w}\|^{2} \mathrm{dV}$,

where $\Omega$ is the domain representing the probed sample.

When numerically solving the quasi-static poroelastic equations (eqs (4)-(7)), the computational domain $\Omega$ is discretized in $n_{x} \times n_{y}$ rectangular cells $\Omega_{k l}$ of area $\delta^{2}$. At each cell $\Omega_{k l}$, the relative fluid displacement $(\boldsymbol{w})_{k l}$ is determined and, thus, the average dissipated power at this location is given by

$\left\langle\Delta \mathcal{P}_{k l}(\omega)\right\rangle=\frac{\omega^{2}}{2}\left(\frac{\eta}{\kappa}\|\boldsymbol{w}\|^{2}\right)_{k l} \delta^{2}$.

The overall average dissipated power throughout the sample is obtained by adding up the values of all cells

$\langle\Delta \mathcal{P}(\omega)\rangle=\sum_{k=1}^{n_{x}} \sum_{l=1}^{n_{y}}\left\langle\Delta \mathcal{P}_{k l}(\omega)\right\rangle$.

\subsubsection{Strain energy}

In order to employ eqs (2) and (3) to compute seismic attenuation, an estimation of the peak strain energy and of the average strain energy stored in the sample are needed. For a monochromatic wave of angular frequency $\omega$, the strain energy stored in cell $\Omega_{k l}$ as a function of time is given by (Biot 1941)

$\left.\mathcal{W}_{k l}(t)\right|_{\omega}=\frac{1}{2}\left(\hat{\boldsymbol{\sigma}}: \hat{\boldsymbol{\epsilon}}+\hat{p}_{f} \hat{\zeta}\right)_{k l} \delta^{2}$,

where $\hat{\boldsymbol{\sigma}}: \hat{\boldsymbol{\epsilon}}=\sum_{i} \sum_{j} \hat{\sigma}_{i j} \hat{\epsilon}_{i j}$ denotes the double dot product of the corresponding tensors. The values of the components of the stress and strain tensors, as well as the fluid pressure and change in the fluid content, are computed using the constitutive equations once the solid and relative fluid displacements, resulting from the applied oscillatory relaxation test, are obtained.
To compute the peak strain energy, we sum up the corresponding values of $\left.\mathcal{W}_{k l}(t)\right|_{\omega}$ over the discretized rock sample and determine the maximum in the time domain

$\mathcal{W}_{\max }(\omega)=\max _{0 \leq t \leq \frac{2 \pi}{\omega}}\left\{\left.\sum_{k=1}^{n_{x}} \sum_{l=1}^{n_{y}} \mathcal{W}_{k l}(t)\right|_{\omega}\right\}$.

Conversely, to estimate the average strain energy over one wave cycle, we again make use of averaging properties of complex-valued time-harmonic variables. As a result, the average strain energy over one wave cycle in a given cell $\Omega_{k l}$ can be expressed as

$\left\langle\mathcal{W}_{k l}(\omega)\right\rangle=\frac{1}{4} \Re\left\{\sigma: \epsilon^{*}+p_{f} \zeta^{*}\right\}_{k l} \delta^{2}$,

where $*$ denotes the complex conjugate.

The average strain energy for the entire sample is then given by

$\langle\mathcal{W}(\omega)\rangle=\sum_{k=1}^{n_{x}} \sum_{l=1}^{n_{y}}\left\langle\mathcal{W}_{k l}(\omega)\right\rangle$.

\subsubsection{Global and local seismic attenuation}

Once the solid and relative fluid displacement fields resulting from the numerical oscillatory relaxation experiments are computed, the implementation of the energy-based approach to quantify the global seismic attenuation is straightforward. Based on eqs (2) and (14), we have

$\frac{1}{Q^{\mathcal{M}}(\omega)}=\frac{\sum_{k=1}^{n_{x}} \sum_{l=1}^{n_{y}}\left\langle\Delta \mathcal{P}_{k l}(\omega)\right\rangle}{\omega \mathcal{W}_{\max }(\omega)}$,

with $\left\langle\Delta \mathcal{P}_{k l}(\omega)\right\rangle$ and $\mathcal{W}_{\max }(\omega)$ given by eqs (13) and (16), respectively. Conversely, considering eqs (3) and (14), we have

$\frac{1}{Q^{\mathcal{A}}(\omega)}=\frac{\sum_{k=1}^{n_{x}} \sum_{l=1}^{n_{y}}\left\langle\Delta \mathcal{P}_{k l}(\omega)\right\rangle}{2 \omega\langle\mathcal{W}(\omega)\rangle}$,

where $\langle\mathcal{W}(\omega)\rangle$ is computed using eq. (18).

As indicated by eq. (20), it is possible to define, for a given frequency $\omega$, the local contribution to seismic attenuation, which is controlled by the average power loss $\left\langle\Delta \mathcal{P}_{k l}(\omega)\right\rangle$ associated with the computational cell $\Omega_{k l}$. That is, eq. (20) can be rewritten as

$\frac{1}{Q^{\mathcal{A}}(\omega)}=\sum_{k=1}^{n_{x}} \sum_{l=1}^{n_{y}} q_{k l}^{-1}(\omega) \delta^{2}$

where $q_{k l}^{-1}(\omega)=\left\langle\Delta \mathcal{P}_{k l}(\omega)\right\rangle /\left(2 \delta^{2} \omega\langle\mathcal{W}(\omega)\rangle\right)$ is the local contribution of the cell $\Omega_{k l}$ to the global seismic attenuation of the probed sample per unit area. This parameter permits to directly observe how the various heterogeneities of the sample govern the overall energy dissipation process. These local attenuation fields, which cannot be provided by approaches based on the DEVM assumption, therefore constitute a very interesting and important feature of the proposed energy-based approach. It is worthwhile to note here that eq. (19) could also be used to define a local contribution to attenuation. This alternative approach, however, would simply imply a rescaling of the local contribution fields defined in eq. (21) and, hence, it is not considered in this work.

\subsection{DEVM approach to quantify seismic attenuation}

The DEVM approach and the energy-based procedure outlined above rely on different assumptions for estimating seismic attenuation. Here, the key premise is the assumption that the rock sample can be represented by a homogeneous viscoelastic solid. Thus, the 
equivalent complex-valued frequency-dependent plane-wave and shear moduli can be obtained by considering the ratios of certain components of the average stress and strain tensors resulting from corresponding numerical relaxation experiments.

In the case of the equivalent plane-wave modulus, it is given by

$\bar{M}_{c}(\omega)=\frac{\overline{\sigma_{y y}}(\omega)}{\overline{\epsilon_{y y}}(\omega)}$,

where $\overline{\sigma_{y y}}(\omega)$ and $\overline{\epsilon_{y y}}(\omega)$ denote the averages over the sample's volume of the vertical components of the stress and strain fields resulting from the numerical relaxation test illustrated in Fig. 1(a).

The equivalent shear modulus is given by

$\bar{\mu}_{c}(\omega)=\frac{1}{2} \frac{\overline{\sigma_{x y}}(\omega)}{\overline{\epsilon_{x y}}(\omega)}$,

where $\overline{\sigma_{x y}}(\omega)$ and $\overline{\epsilon_{x y}}(\omega)$ are the averages over the sample's volume of the non-diagonal components of the stress and strain tensors in response to the numerical oscillatory test illustrated in Fig. 1(b).

Finally, the inverse quality factors are derived from the estimated dynamic moduli using eq. (1). Hence, through approaches based on the DEVM assumption, it is possible to obtain estimations of the overall seismic attenuation experienced by the probed sample, while individual contributions of the heterogeneities cannot be assessed.

\section{GLOBAL AND LOCAL ATTENUATION A NALYSIS}

To study the relation between the energy-based and the DEVM approaches, we compute the corresponding attenuation estimates for a set of pertinent scenarios, namely, patchy saturation and fractured rocks. In addition, we obtain the local contributions to the overall attenuation in each case, which allows to explore the WIFF processes that take place within the corresponding samples.

\subsection{Stochastic patchy saturation model}

In the following, we explore the case of a homogeneous rock frame containing a spatially heterogeneous distribution of two immiscible fluids having very different compressibilities. To this end, we consider a square rock sample of $1 \mathrm{~m}$ sidelength containing a spatially variable $\mathrm{CO}_{2}$-brine distribution in the form of irregular patches fully saturated with $\mathrm{CO}_{2}$ and zones fully saturated with brine. The physical properties of the dry rock, which correspond to those of a poorly consolidated sandstone (Utsira Sand), as well as those of the pore fluids are given in Table 1.

To generate a highly irregular field to be associated with a binary distribution of fluids, we use a von-Karman-type spectral density function of the form

$S_{d}\left(k_{x}, k_{y}\right)=S_{0}\left(1+k_{x}^{2} a_{x}^{2}+k_{y}^{2} a_{y}^{2}\right)^{-(H+E / 2)}$,

Table 1. Physical properties of the solid materials and pore fluids employed in the numerical simulations. Adopted from Rubino et al. $(2011,2014)$.

\begin{tabular}{lccccc}
\hline & Utsira Sand & Sandstone 1 & Brine & $\mathrm{CO}_{2}$ \\
\hline$K_{s}$ & $36.9 \mathrm{GPa}$ & $37 \mathrm{GPa}$ & $K_{f}$ & $2.3 \mathrm{GPa}$ & $0.0229 \mathrm{GPa}$ \\
$\rho_{s}$ & $2650 \mathrm{~kg} \mathrm{~m}^{-3}$ & $2650 \mathrm{~kg} \mathrm{~m}^{-3}$ & $\rho_{f}$ & $1090 \mathrm{~kg} \mathrm{~m}^{-3}$ & $693 \mathrm{~kg} \mathrm{~m}^{-3}$ \\
$\phi$ & 0.37 & 0.1 & $\eta$ & $0.001 \mathrm{~Pa} \mathrm{~s}$ & $1.56 \times 10^{-5} \mathrm{~Pa} \mathrm{~s}$ \\
$K_{m}$ & $2.68 \mathrm{GPa}$ & $26 \mathrm{GPa}$ & & & \\
$\mu_{m}$ & $0.857 \mathrm{GPa}$ & $31 \mathrm{GPa}$ & & & \\
$\kappa$ & $1 \mathrm{D}$ & $10^{-5} \mathrm{D}$ & & & \\
\hline
\end{tabular}

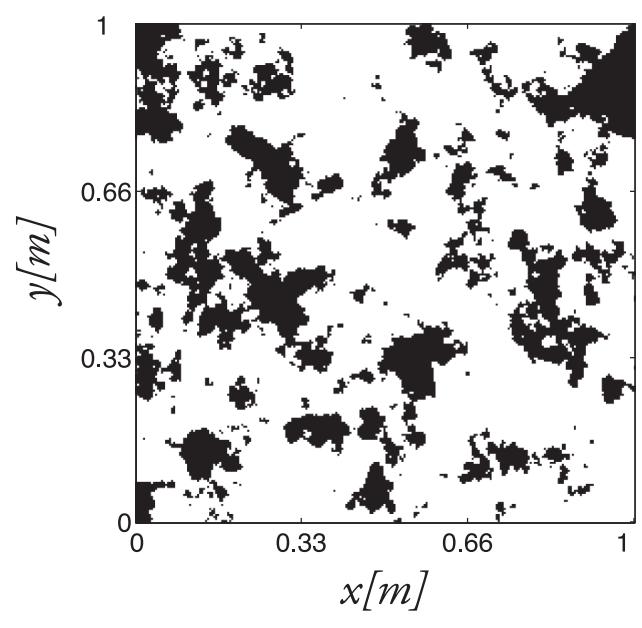

Figure 2. Patchy saturation pattern of $\mathrm{CO}_{2}$ (black regions) and brine (white regions) obtained from a band-limited scale-invariant von-Karman-type distribution through thresholding. The overall $\mathrm{CO}_{2}$ saturation is 0.2 . Please note that the rock matrix is homogeneous.

where $k_{x}$ and $k_{y}$ are the horizontal and vertical wavenumbers, $a_{x}$ and $a_{y}$ are the horizontal and vertical correlation lengths, $S_{0}$ is a normalization constant and $E$ is the Euclidean dimension. The parameter $H$ varies between 0 and 1 and determines the complexity of the considered stochastic process (e.g. Tronicke \& Holliger 2005). This expression corresponds to a band-limited scale-invariant stochastic process with a Hausdorff fractal dimension $D_{H}=E+1-H$. To obtain the fluid distribution from eq. (24), we assign to each grid cell $\Omega_{k l}$ composing the computational domain a pseudo-random number drawn from a uniform distribution. Next, we Fourier transform this field to the spatial wavenumber domain and filter its amplitude spectrum using eq. (24). We transform the result back to the spatial domain and obtain a distribution of random numbers in the form of a $n_{x} \times n_{y}$ matrix. We then use a lineal transformation to map these numbers to values between 0 and 1 . The binary fluid distribution of interest is obtained through thresholding.

Fig. 2 shows the particular fluid distribution obtained following the described procedure and selected for the analysis, which corresponds to a spatially isotropic correlation length of $3.6 \mathrm{~cm}, H=0.8$ and a threshold value of 0.41 . It is important to remark here that, despite its simplicity, binary gas-water saturation patterns in homogeneous dry frames have been considered in the past by several authors to model the seismic response of patchy-saturated media (e.g. White 1975; Dutta \& Odé 1979a; Johnson 2001; Norris 1993; Rubino et al. 2009; Masson \& Pride 2011). Please also note that, as Biot's (1941) equations consider the presence of single-phase pore fluids, some phenomena, such as capillary pressure effects and dissolution, remain unaccounted for in our numerical simulations.

\subsubsection{Attenuation analysis}

Fig. 3 shows the inverse quality factors as functions of frequency for the different approaches considered. We observe that the attenuation curve corresponding to the DEVM assumption $Q_{p}^{\mathcal{D}}$ (red line) is in very good agreement with the one obtained using the energy-based approach $Q_{p}^{\mathcal{A}}$ (dashed blue line). However, the values of the quality factor in the vicinity of the peak are outside the range $Q_{p}^{\mathcal{M}} \gtrsim 10$ where the assumption $\mathcal{W}_{\max } \simeq 2\langle\mathcal{W}\rangle$ is valid for linear viscoelastic solids. We observe that, in this situation, there are discrepancies between $Q_{p}^{\mathcal{M}}$ (solid green line) and the other attenuation 


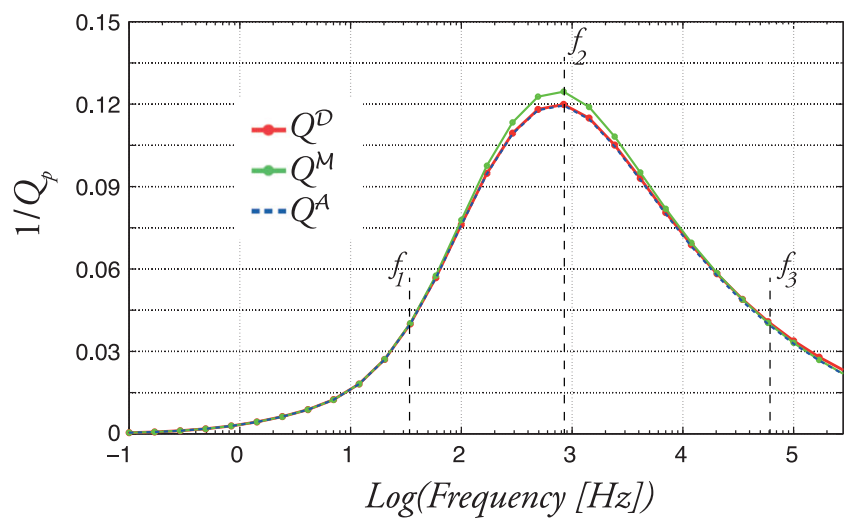

Figure 3. Inverse quality factor as a function of frequency for a homogeneous rock sample containing the fluid distribution shown in Fig. 2. The different curves correspond to the three approaches presented in eqs (1)-(3).

estimates, with maximum relative difference of $\sim 5$ percent. Although not shown here for brevity, in presence of other geometrical characteristics for the gas patches, such as in the case of White's periodically layered medium, the relative differences can reach values of up to $\sim 10$ per cent for overall gas saturations of $S_{g} \sim 10$ per cent and the dry-frame and fluid properties considered in this example. These results thus indicate that the attenuation estimates based on the DEVM assumption are in very good agreement with eq. (3) and that the relative differences with eq. (2) are significant only in presence of extreme compressibility contrasts or, equivalently, in the case of very high attenuation levels. These behaviours are indeed similar to the ones expected for linear viscoelastic solids and thus verify that the DEVM assumption is in accordance with the energybased procedure in presence of strong contrasts of the compressibilities of the saturating pore fluids. Please note that the attenuation curves are non-symmetric, as previously shown by other authors (e.g. Müller \& Rothert 2006), which indicates that the DEVM does not corresponds to a simple rheological model, such as Maxwell or Zener models.

The energy-based approach allows to analyse the WIFF process in terms of the local contribution to attenuation $q_{k l}^{-1}(\omega)$ (eq. 21). Fig. 4 shows the spatial distribution of $q_{k l}^{-1}$ for the three frequencies indicated in Fig. 3, that is, $f_{1}=34.5 \mathrm{~Hz}, f_{2}=837.7 \mathrm{~Hz}$ and $f_{3}=5.8$ $\times 10^{4} \mathrm{~Hz}$. Thereby, $f_{2}$ is the frequency at which attenuation peaks, whereas $f_{1}$ and $f_{3}$ are chosen so that $Q_{p}\left(f_{1}\right)=Q_{p}\left(f_{3}\right)$. Please note that, for each frequency, by adding up the values of $q_{k l}^{-1}$ in Fig. 4 over the entire rock sample and multiplying the result by the cell area $\delta^{2}$, the corresponding value of the overall attenuation curve shown in Fig. 3 is obtained. An interesting feature illustrated by Fig. 4 is that, regardless of the frequency, the main contribution to attenuation occurs within the brine-saturated regions, particularly in the vicinity of the $\mathrm{CO}_{2}$ patches. This is expected as the largest pressure gradients are induced at the $\mathrm{CO}_{2}$-brine interfaces and the subsequent relative fluid-solid motion results in larger dissipation in the more viscous fluid phase (see eq. 13).

A key parameter to understand the changes of the distributions of $q_{k l}^{-1}(\omega)$ with frequency is the diffusion length, which quantifies the spatial scales measured from the interfaces at which dissipation due to WIFF is significant. The diffusion length is given by (Müller et al. 2010)

$L_{d}=\sqrt{D / \omega}$,

where $D$ is the diffusivity of the medium, a parameter that can be computed from the poroelastic properties. For very low
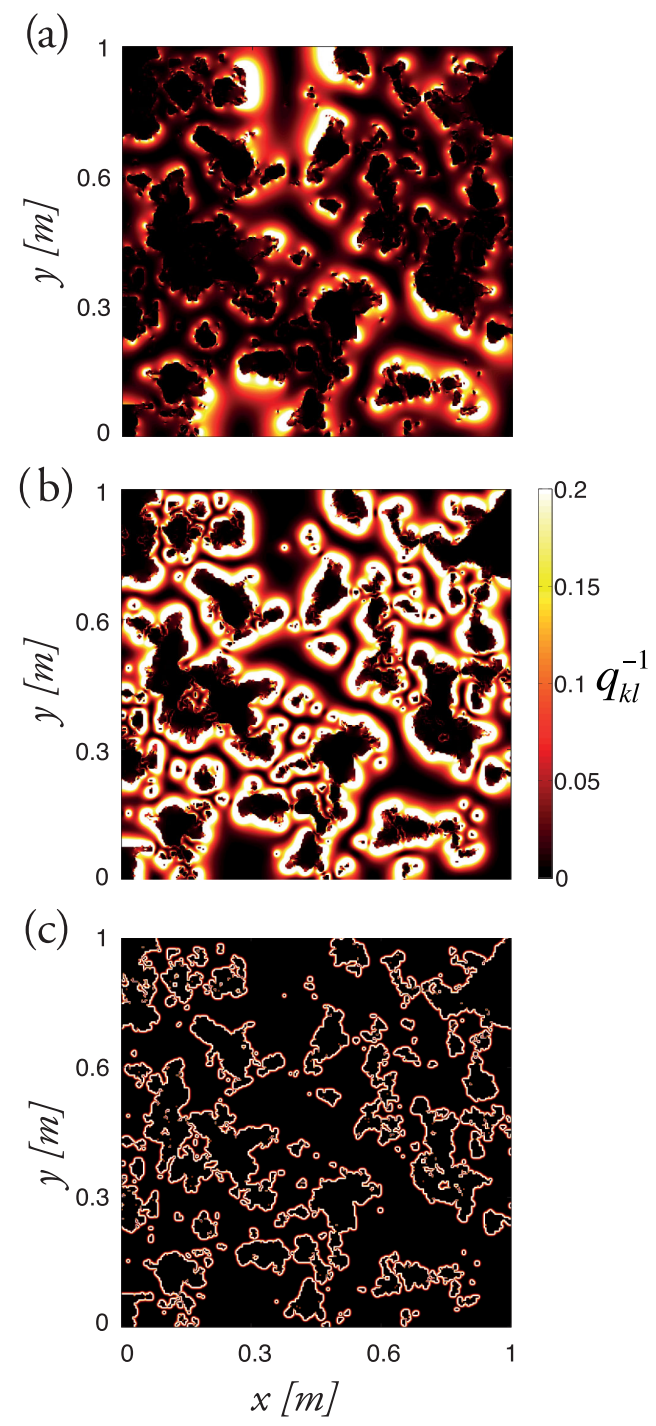

Figure 4. Spatial distribution of $q_{k l}^{-1}\left[\mathrm{~m}^{-2}\right]$, for the sample shown in Fig. 2. The panels correspond to three frequencies of (a) $f_{1}=34.5 \mathrm{~Hz}$, (b) $f_{2}=$ $837.7 \mathrm{~Hz}$ and (c) $f_{3}=5.8 \times 10^{4} \mathrm{~Hz}$.

frequencies, the diffusion length is much larger than the sizes of the heterogeneities and, hence, there will be enough time during each oscillatory half-cycle for the fluid pressure to equilibrate at a common value. Correspondingly, this low-frequency regime represents a relaxed state. Conversely, for very high frequencies, there is no time for communication between the pore fluid of the different regions composing the probed medium and, thus, this high-frequency regime is associated with an unrelaxed state. For a frequency $f_{c}$, the diffusion lengths are comparable to the characteristic size of the heterogeneities and significant fluid flow and energy dissipation can be induced by the passing wave. Thus, this characteristic frequency $f_{c}$ is also associated with the location of the attenuation peak.

At the frequency $f_{1}$, the $q_{k l}^{-1}$ fringes are unevenly distributed and vanish almost entirely around certain $\mathrm{CO}_{2}$ patches. This is due to the fact that at this low frequency the diffusion length $L_{d}$ can exceed the distance separating some neighbouring $\mathrm{CO}_{2}$ patches. Such regions then effectively behave like one large patch and in their interior the local contributions $q_{k l}^{-1}$ are essentially negligible. Comparing Figs 2 and 4(a) illustrates that, for this frequency, the regions that significantly contribute to the overall attenuation are 
related to the brine-saturated patches with bigger characteristic size that are relatively free of small $\mathrm{CO}_{2}$ patches. At the frequencies $f_{2}$ and $f_{3}$, local dissipation contributions $q_{k l}^{-1}$ can be observed around all $\mathrm{CO}_{2}$ patches. However, these $q_{k l}^{-1}$ fringes are larger for $f=f_{2}$ and therefore the overall attenuation is also higher, that is, $Q_{p}^{-1}\left(f_{2}\right)>$ $Q_{p}^{-1}\left(f_{3}\right)$.

As the diffusion length scales as $1 / \sqrt{f}$ (eq. 25), one expects that the spatial extent over which the local dissipation contributions $q_{k l}^{-1}$ assume significant values, shrinks to the immediate vicinity of the $\mathrm{CO}_{2}$ patches when the frequency increases. This behaviour prevails when the frequency increases from $f_{2}$ to $f_{3}$ (Figs 4 (b) and (c)). However, Figs 4(a) and (b) indicate that for the brine-saturated regions surrounding $\mathrm{CO}_{2}$ patches having relatively small characteristic size, the spatial extent over which energy is dissipated broadens as the frequency increases from $f_{1}$ to $f_{2}$. This evidences the interaction between patches in the energy dissipation process. Also, it is worthwhile to note that even though the local attenuation contributions $q_{k l}^{-1}$ shown in Figs 4(a) and (c) are very different, the overall attenuation at these two frequencies is the same (Fig. 3). The advantage of the local attenuation analysis is that these characteristics can be identified and analysed, which eventually may allow us to better understand certain aspects of the energy dissipation process, such as, for example, the interaction between pressure relaxation processes initiated at neighbouring patches.

\subsection{Fractured media}

The presence of fractures may produce very high levels of attenuation due to WIFF as a consequence of the strong compressibility contrasts typically observed with regard to their embedding matrix (e.g. Rubino et al. 2014). To study the relation between the energybased and the DEVM approaches for this scenario, we compare the corresponding attenuation estimates. In this case, we compute $P$ and $S$-wave inverse quality factors for the vertical direction of wave propagation by means of the previously described numerical oscillatory relaxation tests (Fig. 1) applied to a representative square rock sample of $2.75 \mathrm{~cm}$ sidelength containing a fracture with an inclination of $45^{\circ}$ (Fig. 5).

In the given context, it is convenient to represent fractures as part of a poroelastic continuum (Brajanovsky et al. 2005; Nakagawa \& Schoenberg 2007; Gurevich et al. 2009; Rubino et al. 2014). We model the fracture as a very thin elliptical porous medium of very high porosity and permeability embedded in relatively stiff sandstone with physical properties given in Table 1 (Sandstone 1). We assume that, at the grain level, the solid material properties of the fracture and those of the embedding matrix are identical. Choosing a maximum fracture aperture $h$ of $0.033 \mathrm{~cm}$ and following Nakagawa \& Schoenberg (2007), we obtain $K_{m}^{f}=0.02 \mathrm{GPa}$ and $\mu_{m}^{f}=0.01 \mathrm{GPa}$ for the dry-frame elastic properties of the fracture (Rubino et al. 2014). For the porous material filling the fracture, we assume a porosity $\phi^{f}$ of 0.5 and a permeability $\kappa^{f}$ of $100 \mathrm{D}$. Both the fracture and the embedding matrix are fully saturated with brine (Table 1).

\subsubsection{Attenuation analysis}

Fig. 6 shows a comparison of the inverse $P$ - and $S$-wave quality factors obtained using the energy-based and the DEVM approaches. In the case of the $P$-wave inverse quality factor (Fig. 6a), the three curves are very similar throughout the pertinent frequency range. Conversely, in the case of the inverse $S$-wave quality factor (Fig. 6b),

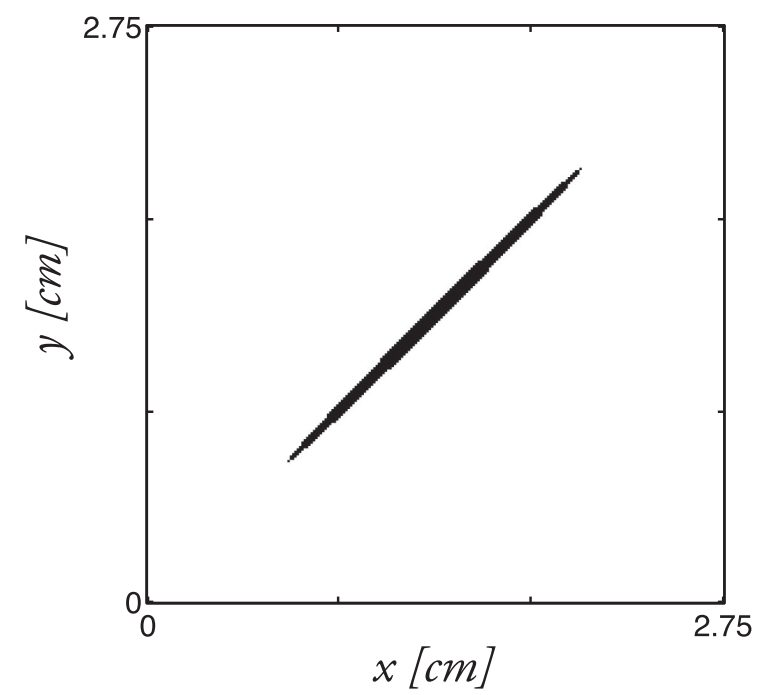

Figure 5. Rock sample containing a $45^{\circ}$-inclined elliptical fracture with a maximum aperture $h$ of $0.033 \mathrm{~cm}$. The background (white region) corresponds to a low-porosity sandstone and the fracture (black region) is modelled as a highly porous and permeable medium. The sample is fully saturated with brine.

(a)
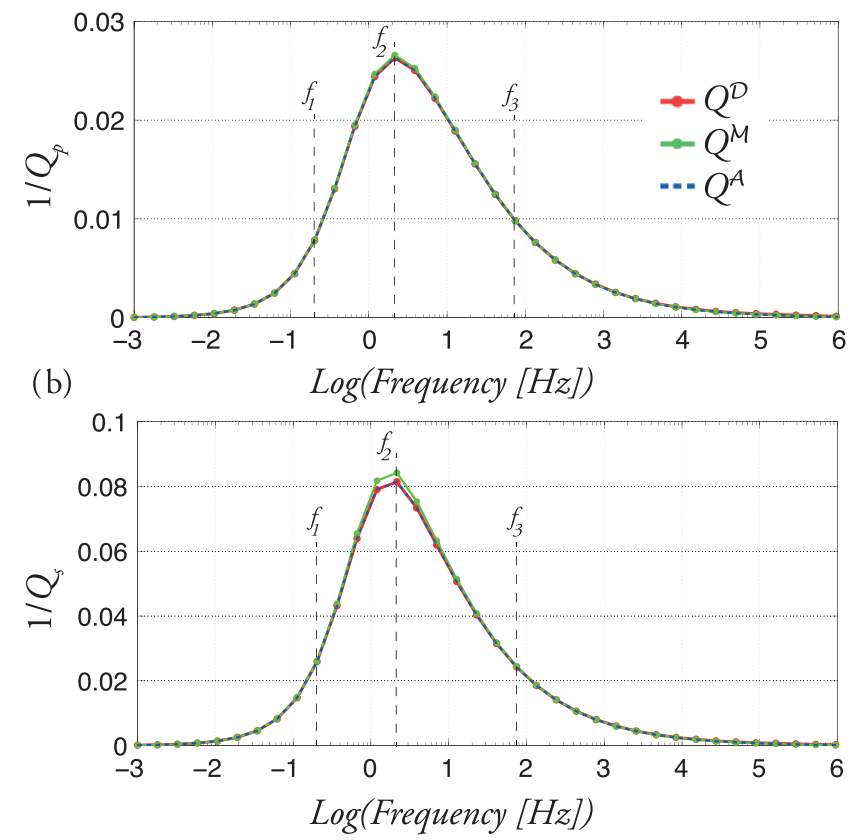

Figure 6. Inverse quality factor as a function of frequency for the sample shown in Fig. 5 for vertically propagating (a) $P$ and (b) $S$ waves . The curves correspond to the different approaches presented in eqs (1)-(3).

we observe that $Q_{s}^{\mathcal{M}}$ slightly deviates with respect to $Q_{s}^{\mathcal{D}}$ and $Q_{s}^{\mathcal{A}}$ when $Q_{s}^{\mathcal{M}} \lesssim 10$. These results, which are similar to the ones expected for linear viscoelastic solids, thus illustrate that the DEVM assumption is in accordance with the energy-based estimate even in the presence of very strong contrasts of the dry-frame elastic properties.

In order to gain further insight into the physics of WIFF in presence of fractures, we now focus on the local contributions to attenuation per unit area $q_{k l}^{-1}$ for both $P$ and $S$ waves. Figs 7 and 8 display these contributions at the frequencies indicated in the curves shown 
(a)

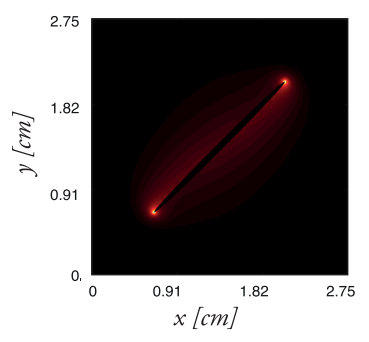

(b)

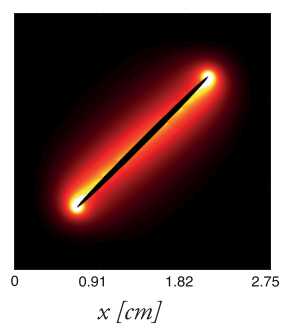

(c)

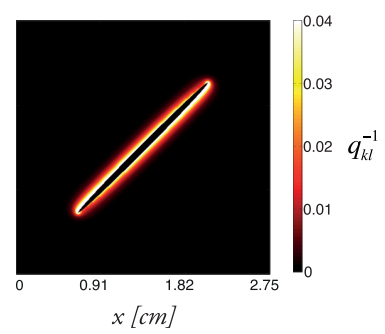

Figure 7. Spatial distribution of $q_{k l}^{-1}\left[\mathrm{~m}^{-2}\right]$, for the sample shown in Fig. 5 for vertically propagating $P$ waves with frequencies of (a) $f_{1}=0.2 \mathrm{~Hz}$, (b) $f_{2}=$ $2.15 \mathrm{~Hz}$ and (c) $f_{3}=74.44 \mathrm{~Hz}$.

(a)

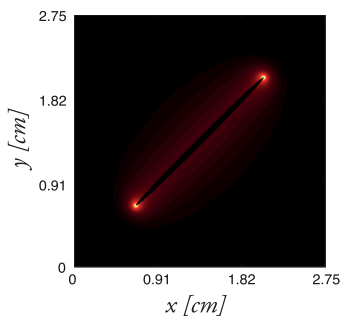

(b)

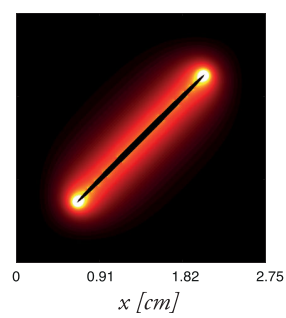

(c)

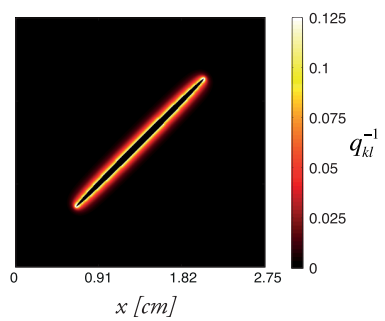

Figure 8. Spatial distribution of $q_{k l}^{-1}\left[\mathrm{~m}^{-2}\right]$, for the sample shown in Fig. 5 for vertically propagating $S$ waves with frequencies of (a) $f_{1}=0.2 \mathrm{~Hz}$, (b) $f_{2}=$ $2.15 \mathrm{~Hz}$ and (c) $f_{3}=74.44 \mathrm{~Hz}$

in Fig. 6: $f_{1}=0.2 \mathrm{~Hz}$, the peak frequency $f_{2}=2.15 \mathrm{~Hz}$ and $f_{3}=$ $74.44 \mathrm{~Hz}$. We observe that for both $P$ and $S$ waves most of the energy dissipation takes place in the background material close to the fracture. This is expected, as the fracture material is more compliant than the embedding matrix and, therefore, during the positive cycle of the applied displacement, fluid flow takes place from the fracture into the background. According to eq. (13), energy dissipation is inversely proportional to permeability. This, in turn, implies that seismic attenuation is mainly produced in the regions of low permeability affected by WIFF, that is, in the background material in the vicinity of the fracture. It is also interesting to observe that, while the local contribution to attenuation exhibits a $z$-shaped pattern for $P$ waves (Fig. 7b), the corresponding pattern for $S$ waves is rather symmetrical with respect to the fracture (Fig. 8 b).

It can also be noted that, both for $P$ and $S$ waves, the highest contributions to attenuation prevail in the immediate vicinity of the fracture tips (Figs 7 and 8). This is an interesting feature that may have important implications with respect to the frequency range affected by WIFF. Indeed, it has been formulated in several classical papers reviewed by Müller et al. (2010) that the attenuation peak occurs at a frequency $f_{c}$ for which the diffusion length $L_{d}$ is comparable with the characteristic size of the heterogeneities. This is true for heterogeneities for which fluid flow takes place regularly along their boundaries, such as in the case of patchy saturation. However, the fact that for fractured media most of the energy dissipation is concentrated at the fracture tips (Figs 7 and 8) indicates that this relationship between diffusion length, characteristic sizes of the heterogeneities and frequency of the attenuation peak, is not expected to hold for such scenarios.

\section{CONCLUSIONS}

We have proposed a novel methodology to compute seismic attenuation due to WIFF at the mesoscopic scale in heterogeneous poroelastic media. It is based on the direct calculation of the amount of dissipated power and stored strain energy associated with a passing seismic wavefield through the application of appropriate numerical oscillatory relaxation tests on representative rock samples.

Our approach allowed us to verify that the attenuation estimates based on the widely used DEVM assumption are in agreement with those obtained through a methodology fully rooted in the theoretical framework of poroelasticity. This is true irrespective of strong contrasts of fluid compressibilities and dry-frame elastic moduli, characteristic of patchy saturation and fractured media, respectively. In all cases considered in this work, the inverse quality factor defined in terms of the average power loss and the average strain energy is identical with that obtained based on the DEVM assumption. Using the peak strain energy to compute the inverse quality factor yields slight differences for high attenuation values. These behaviours, which are similar to those expected for linear viscoelastic solids, support the validity of the common assumption that the upscaled counterpart of a poroelastic sample having heterogeneities in the mesoscopic scale range can be represented by an equivalent homogeneous viscoelastic solid.

A key feature of the proposed energy-based approach is that, unlike the methodologies based on the DEVM assumption, it allows us to assess the local contributions to the overall seismic attenuation. This additional information can be used to identify the regions mainly controlling the energy dissipation process, such as the tips of a fracture. It can also be used to study the interaction of pressure diffusion processes initiated at neighbouring heterogeneities. Therefore, this new approach has the potential of fundamentally improving our current understanding of the WIFF attenuation mechanism.

\section{ACKNOWLEDGEMENTS}

This work was supported in part by a grant from the Swiss Government through the Federal Commission for Scholarships for Foreign Students (FCS). The authors thank Eva Caspari for enlightening 
discussions and two anonymous reviewers for lucid comments and helpful suggestions.

\section{REFERENCES}

Aki, K. \& Richards, P.G., 1980. Quantitative Seismology: Theory and Methods, W.H. Freeman.

Biot, M.A., 1941. General theory of three-dimensional consolidation, $J$. appl. Phys., 12, 155-164.

Biot, M.A., 1962. Mechanics of deformation and acoustic propagation in porous media, J. appl. Phys., 33(4), 1482-1498.

Borcherdt, R.D., 2009. Viscoelastic Waves in Layered Media, Cambridge Univ. Press.

Bourbié, T., Coussy, O. \& Zinszner, B., 1987. Acoustics of Porous Media, Institut Français du Pétrole Publications.

Brajanovsky, M., Gurevich, B. \& Schoenberg, M., 2005. A model for $P$ wave attenuation and dispersion in a porous medium permeated by aligned fractures, Geophys. J. Int., 163, 372-384.

Dutta, N.C. \& Odé, H., 1979a. Attenuation and dispersion of compressional waves in fluid-filled rocks with partial gas saturation (White model) —Part I: Biot theory, Geophysics, 44, 1777-1788.

Dutta, N.C. \& Odé, H., 1979b. Attenuation and dispersion of compressional waves in fluid-filled rocks with partial gas saturation (White model) —Part II: Results, Geophysics, 44, 1789-1805.

Gurevich, B., Brajanovsky, M., Galvin, R.J., Müller, T.M. \& Toms-Stewart, J., 2009. $P$-wave dispersion and attenuation in fractured and porous reservoirs - poroelasticity approach, Geophys. Prospect., 57, 225-237.

Hashin, Z., 1964. Theory of mechanical behavior of heterogeneous media, Appl. Mech. Rev., 17, 1-9.

Hill, R., 1963. Elastic properties of reinforced solids: some theoretical principles, J. Mech. Phys. Solids, 11, 357-372.

Hill, R., 1972. On constitutive maco-variables for heterogeneous solids at finite strain, Proc. R. Soc. Lond. A, 326, 131-147.

Jänicke, R., Quintal, B. \& Steeb, H., 2015. Numerical homogenization of mesoscopic loss in poroelastic media, Eur. J. Mech. A Solids, 49, 382-395.

Johnson, D.L., 2001. Theory of frequency dependent acoustics in patchysaturated porous media, J. acoust. Soc. Am., 110, 682-694.

Masson, T.J. \& Pride, S.R., 2007. Poroelastic finite difference modeling of seismic attenuation and dispersion due to mesoscopic-scale heterogeneity, J. geophys. Res., 112, B03204, doi:10.1029/2006JB004592.

Masson, Y.J. \& Pride, S.R., 2011. Seismic attenuation due to patchy saturation, J. geophys. Res., 116, doi:10.1029/2010JB007983.

Masson, Y.J. \& Pride, S.R., 2014. On the correlation between material structure and seismic attenuation anisotropy in porous media, J. geophys. Res., 119, B03206, doi:10.1002/2013JB010798.

Müller, T.M. \& Rothert, E., 2006. Seismic attenuation due to wave-induced flow: why Q in random structures scales differently, Geophys. Res. Lett., 33, doi:10.1029/2006GL026789.

Müller, T.M., Gurevich, B. \& Lebedev, M., 2010. Seismic wave attenuation and dispersion resulting from wave-induced flow in porous rocks-a review, Geophysics, 75, 147-163.

Nakagawa, S. \& Schoenberg, A., 2007. Poroelastic modeling of seismic boundary conditions across a fracture, J. acoust. Soc. Am., 122, 831-847.

Nedelec, J.C., 1980. Mixed finite elements in $\mathrm{R}^{3}$, Numer. Math., 35, 315341.

Norris, A.N., 1993. Low-frequency dispersion and attenuation in partially saturated rocks, J. acoust. Soc. Am., 94, 359-370.

O'Connell, R.J. \& Budiansky, B., 1978. Measures of dissipation in viscoelastic media, Geophys. Res. Lett., 5, 5-8.

Picotti, S., Carcione, J.M., Rubino, J.G., Santos, J.E. \& Cavallini, F., 2010. A viscoelastic representation of wave attenuation in porous media, Comput. Geosci., 36, 44-53.

Quintal, B., Steeb, H., Frenher, M. \& Schmalholz, S.M., 2011. Quasi-static finite element modeling of seismic attenuation and dispersion due to wave- induced fluid flow in poroelastic media, J. geophys. Res., 116, B01201, doi:10.1029/2010JB007475.

Raviart, P. \& Thomas, J., 1977. Mixed finite element method for 2nd order elliptic problems, Lect. Notes Math., 606, 292-315.

Rubino, J.G., Ravazzoli, C.L. \& Santos, J.E., 2006. Reflection and transmission of waves in composite porous media: a quantification of energy conversions involving slow waves, J. acoust. Soc. Am., 120(5), 24252436.

Rubino, J.G., Ravazzoli, C.L. \& Santos, J.E., 2009. Equivalent viscoelastic solids for heterogeneous fluid-saturated porous rocks, Geophysics, 74, N1-N13.

Rubino, J.G., Velis, D.R. \& Sacchi, M.D., 2011. Numerical analysis of waveinduced fluid flow effects on seismic data: application to monitoring of $\mathrm{CO}_{2}$ storage at the Sleipner field, J. geophys. Res., 116, 1088-1102.

Rubino, J.G., Müller, T.M., Guarracino, L., Milani, M. \& Holliger, K., 2014. Seismoacustic signatures of fracture connectivity, J. geophys. Res., 119, 2252-2271

Sidler, R., Rubino, J.G. \& Holliger, K., 2013. Quantitative comparison between simulations of seismic wave propagation in heterogeneous poroelastic media and equivalent visco-elastic solids for marine-type environments, Geophys. J. Int., 193, 463-474.

Spencer, J.W., 1981. Stress relaxations at low frequencies in fluid-saturated rocks: attenuation and modulus dispersion, J. geophys. Res., 86, 18031812.

Takei, Y., Fujisawa, K. \& McCarthy, C., 2011. Experimental study of attenuation and dispersion over a broad frequency range: 1 . The apparatus, $J$. geophys. Res., 115, B09204, doi:10.1029/2011JB008382.

Tisato, N. \& Quintal, B., 2013. Measurements of seismic attenuation and transient fluid pressure in partially saturated Berea sandstone: evidence of fluid flow on the mesoscopic scale, Geophys. J. Int., 195, 342-351.

Tronicke, J. \& Holliger, K., 2005. Quantitative integration of hydrogeophysical data: conditional geostatistical simulation for characterizing heterogeneous alluvial aquifers, Geophysics, 70, H1-H10.

Wenzlau, F., Altman, J. \& Müller, T.M., 2010. Anisotropic dispersion and attenuation due to wave-induced fluid flow: quasi-static finite element modeling in poroelastic solids, J. geophys. Res., 115, B07204, doi:10.1029/2009JB006644.

White, J.E., 1975. Computed seismic speeds and attenuation in rocks with partial gas saturation, Geophysics, 40, 224-232.

White, J.E., Mikhaylova, N.G. \& Lyakhovitsky, F.M., 1975. Low-frequency seismic waves in fluid-saturated layered rocks, Phys. Solid Earth, 11, 654-659.

\section{APPENDIX: BOUNDARY CONDITIONS RELATED TO THE NUMERICAL OSCILLATORY RELAXATION TESTS}

To estimate the response of a synthetic rock sample subjected to a numerical oscillatory relaxation test, eqs (4)-(7) are solved using a finite-element procedure under corresponding boundary conditions. Let $\Omega=\left(0, L_{x}\right) \times\left(0, L_{y}\right)$ be a domain that represents the probed sample. In addition, $\Gamma$ is the boundary of $\Omega$, which is given by $\Gamma=$ $\Gamma^{L} \cup \Gamma^{B} \cup \Gamma^{R} \cup \Gamma^{T}$, where

$\Gamma^{L}=\{(x, y) \in \Omega: x=0\}$,

$\Gamma^{R}=\left\{(x, y) \in \Omega: x=L_{x}\right\}$

$\Gamma^{B}=\{(x, y) \in \Omega: y=0\}$,

$\Gamma^{T}=\left\{(x, y) \in \Omega: y=L_{y}\right\}$

Moreover, $\boldsymbol{v}$ denotes the unit outer normal on $\Gamma$ and $\chi$ is a unit tangent so that $\{\boldsymbol{v}, \chi\}$ is an orthonormal system on $\Gamma$. 
10 S.G. Solazzi et al.

To estimate $P$-wave attenuation, we consider the following boundary conditions

$\boldsymbol{u} \cdot \boldsymbol{v}=-\Delta u, \quad(x, y) \in \Gamma^{T}$,

(A5)

$\boldsymbol{u} \cdot \boldsymbol{v}=0, \quad(x, y) \in \Gamma^{L} \cup \Gamma^{R} \cup \Gamma^{B}$,

(A6)

$(\sigma \cdot v)^{\mathrm{T}} \cdot \chi=0, \quad(x, y) \in \Gamma$,

(A7) $\quad(\boldsymbol{\sigma} \cdot \boldsymbol{v})^{\mathrm{T}} \cdot \boldsymbol{v}=0, \quad(x, y) \in \Gamma$,

(A8) $\boldsymbol{w} \cdot \boldsymbol{v}=0, \quad(x, y) \in \Gamma$.

The set of boundary conditions for estimating $S$-wave attenuation is given by

$\boldsymbol{u} \cdot \boldsymbol{\chi}=\Delta u, \quad(x, y) \in \Gamma^{T}$,

(A9)

$\boldsymbol{w} \cdot \boldsymbol{v}=0, \quad(x, y) \in \Gamma$. 